JOURNAL OF

FUNCTION SPACES AND APPLICATIONS

Volume 1, Number 1 (2003), 35-43 (c) 2003, Scientific Horizon http://www.jfsa.net

\title{
On a measure of non-compactness for singular integrals
}

\author{
Alexander Meskhi \\ (Communicated by V. Kokilashvili)
}

1991 Mathematics Subject Classification. 42B20

Keywords and phrases. Singular integrals, Riesz transforms, Hilbert transforms, Cauchy integral, weight.

Abstract. It is proved that there exists no weight pair $(v, w)$ for which a singular integral operator is compact from the weighted Lebesgue space $L_{w}^{p}\left(R^{n}\right)$ to $L_{v}^{p}\left(R^{n}\right)$. Moreover, a measure of non-compactness for this operator is estimated from below. Analogous problems for Cauchy singular integrals defined on Jordan smooth curves are studied.

\section{Introduction}

In this paper we show that there exists no weight pair $(v, w)$ for which the singular integral operator

$$
K f(x)=p \cdot v \cdot \int_{R^{n}} k(x-y) f(y) d y
$$

is compact from the weighted Lebesgue space $L_{w}^{p}\left(R^{n}\right)$ to $L_{v}^{p}\left(R^{n}\right)$. A measure of non-compactness (essential norm)

$$
\|K\|_{\mathcal{K}} \equiv \operatorname{dist}\left\{K, \mathcal{K}\left(L_{w}^{p}\left(R^{n}\right), L_{v}^{p}\left(R^{n}\right)\right)\right\}
$$


for the operator $K$, where $\mathcal{K}\left(L_{w}^{p}\left(R^{n}\right), L_{v}^{p}\left(R^{n}\right)\right)$ is a space of all compact operators acting from $L_{w}^{p}\left(R^{n}\right)$ to $L_{v}^{p}\left(R^{n}\right)$, is estimated from below. We also consider analogous problems for the Cauchy singular operator

$$
S_{\Gamma} f(t)=p \cdot v \cdot \frac{1}{\pi i} \int_{\Gamma} \frac{f(\tau)}{\tau-t} d \tau, t=t(s), 0 \leq s \leq l \leq \infty,
$$

along a smooth Jordan curve $\Gamma$ (of the complex plane) on which the arclength is chosen as a parameter.

The essential norm $\left\|S_{T}\right\|_{\mathcal{K}} \equiv \operatorname{dist}\left\{S_{T}, \mathcal{K}\left(L^{p}(T), L^{p}(T)\right)\right\}$ for the operator $S_{T}$, where $T$ is the unit circle, was calculated in [11-12] for $p=2^{n}$ and $p=\frac{2^{n}}{2^{n}-1}$. In that paper a lower estimate for $\left\|S_{T}\right\|_{\overline{\mathcal{K}}}$ was also derived for all $p \in(1, \infty)$. An upper estimate for $\left\|S_{T}\right\|_{\overline{\mathcal{K}}}, 1<p<\infty$, was obtained in [17]. In the case of weighted Lebesgue spaces with power weights the essential norm of a Cauchy singular integral over Lyapunov curves was calculated in [16]. The case of general Muckenhoupt weights was considered in [8], where it was shown that $\operatorname{dist}\left\{S_{T}, \mathcal{K}\left(L_{w}^{2}(T), L_{w}^{2}(T)\right)\right\}=1$ if and only if $w$ has a vanishing mean oscillation.

\section{Preliminaries}

Let $w$ be a locally integrable almost everywhere positive function (i.e. a weight) on $\Omega$, where $\Omega$ is a domain in $R^{n}$. Denote by $L_{w}^{p}(\Omega)(1<p<\infty)$ the weighted Lebesgue space which is a space of all measurable functions $f: \Omega \rightarrow R$ with finite norm

$$
\|f\|_{L_{w}^{p}(\Omega)}=\left(\int_{\Omega}|f(x)|^{p} w(x) d x\right)^{1 / p}
$$

If $w \equiv 1$, then we denote $L_{w}^{p}(\Omega)$ by $L^{p}(\Omega)$.

Definition 2.1. Let $1<p<\infty$. We say that the weight $w$ belongs to $A_{p}\left(R^{n}\right)$ if

$$
\sup \left(\frac{1}{|B|} \int_{B} w(x) d x\right)\left(\frac{1}{|B|} \int_{B} w^{1-p^{\prime}}(x) d x\right)^{p-1}<\infty, \quad p^{\prime}=\frac{p}{p-1},
$$

where the supremum is taken over all balls $B$ in $R^{n}$ and $|B|$ is a measure of $B$.

We denote

$$
g_{B}:=\frac{1}{|B|} \int_{B}|g(x)| d x
$$

for a measurable function $g$ and a ball $B \subset R^{n}$.

In the sequel we shall assume that there exists a positive constant $c$ such that

$$
\|K f\|_{L^{2}\left(R^{n}\right)} \leq c\|f\|_{L^{2}\left(R^{n}\right)}, \quad f \in C_{0}^{\infty}\left(R^{n}\right) .
$$


We shall also suppose that the kernel $k$ satisfies the following two conditions:

(i) there exists a positive constsnt $A$ such that the inequality

$$
\left|\left(\frac{\partial}{\partial x}\right)^{\alpha} k(x)\right| \leq A|x|^{-n-\alpha}
$$

holds for all $x \in R^{n}, x \neq 0$, and $|\alpha| \leq 1$;

(ii) there exists a positive constant $b$ and an unit vector $u_{0}$ such that

$$
|k(x)| \geq b|x|^{-n}
$$

when $x=\lambda \cdot u_{0}$ with $-\infty<\lambda<+\infty$.

It is easy to see that the Riesz transforms

$$
R_{j} f(x)=\lim _{r \rightarrow 0} \gamma_{n} \int_{R^{n} \backslash B(x, r)} \frac{x_{j}-y_{j}}{|x-y|^{n+1}} f(y) d y, \quad j=1, \ldots, n,
$$

where $x=\left(x_{1}, \ldots, x_{n}\right) \in R^{n}, \gamma_{n}=\Gamma[(n+1) / 2] / \pi^{(n+1) / 2}$, satisfy conditions (2.1)-(2.3). If $n=1$, then $R_{1} f(x)$ is the Hilbert transform defined by

$$
H f(x)=\lim _{\varepsilon \rightarrow 0} \frac{1}{\pi} \int_{|x-y|>\varepsilon} \frac{f(y)}{x-y} d y .
$$

It is known that $H$ is bounded in $L_{w}^{p}(R), 1<p<\infty$, if and only if $w \in A_{p}(R)$ (see [13]). In [2] it was proved that the Calderón- Zygmund singular operator is bounded in $L_{w}^{p}\left(R^{n}\right), 1<p<\infty$, if $w \in A_{p}\left(R^{n}\right)$. The necessity of the condition $w \in A_{p}\left(R^{n}\right)$ for the boundedness of $R_{j}$ was established in [9], p. 417.

Theorem A ([18], Ch. 5, 4.2; 4.6). If conditions (2.1)-(2.2) are satisfied and $w \in A_{p}\left(R^{n}\right)$, then the operator $K$ is bounded in $L_{w}^{p}\left(R^{n}\right)$. Further, if (2.1)-(2.3) hold and $K$ is bounded in $L_{w}^{p}\left(R^{n}\right)$, then $w \in A_{p}\left(R^{n}\right)$.

Finally we note that optimal sufficient conditions on radial weight pairs $(v, w)$ governing the boundedness of the Calderón-Zygmund operators from $L_{w}^{p}\left(R^{n}\right)$ to $L_{v}^{p}\left(R^{n}\right)$ were established in [4]. An analogous problem for singular integrals defined on measure spaces with a quasi-metric and doubling measure (SHT) was solved in [5] (see also [10], Ch. 9 and [6], Ch. 7).

Suppose that $X$ and $Y$ are Banach spaces. We denote by $\mathcal{K}(X, Y)$ the space of all compact linear operators acting from $X$ to $Y$. Let $F_{r}(X, Y)$ be the space of all finite rank operators from $X$ to $Y$.

The following statement is from [3] (Corollary V. 5. 4).

Lemma 2.1. Let $1 \leq p<\infty$. Suppose that $P$ is a bounded linear operator from $X$ to $Y$, where $Y=L^{p}(\Omega)$. Then

$$
\operatorname{dist}\{P, \mathcal{K}(X, Y)\}=\operatorname{dist}\left\{P, F_{r}(X, Y)\right\} \text {. }
$$


In the sequel by $B(x, r)$ will be denoted the ball with center $x$ and radius $r$.

The next statement is similar to Lemma V.5.6 from [3].

Lemma 2.2. Let $P \in F_{r}\left(X, L^{p}(\Omega)\right)$, where $X=L_{w}^{r}(\Omega), 1<r, p<\infty$. Then for any $a \in \Omega$ and $\varepsilon>0$ there exist $R \in F_{r}\left(X, L^{p}(\Omega)\right)$ and a positive number $\alpha$ such that the inequality

$$
\|(P-R) f\|_{L_{p}(\Omega)} \leq \varepsilon\|f\|_{X}
$$

holds and $\operatorname{supp} R f \subset \Omega \backslash B(a, \alpha)$ for all $f \in X$.

Proof. From $P \in F_{r}(X, \Omega)$ it follows that there exist linearly independent functions $u_{j} \in L^{p}(\Omega)$ such that

$$
\operatorname{Pf}(x)=\sum_{j=1}^{N} \beta_{j}(f) u_{j}(x), \quad f \in X,
$$

where $\beta_{j}$ are linear functionals defined on $X$ (i.e., $\beta_{j} \in X^{*}$ ).

On the other hand, there exists a positive constant $c$ for which the inequality

holds.

$$
\sum_{j=1}^{N}\left|\beta_{j}(f)\right| \leq c\|f\|_{X}
$$

Let us choose linearly independent functions $\Phi_{j} \in L^{p}\left(R^{n}\right)$ and real numbers $\alpha_{j}$ such that

$$
\left\|u_{j}-\Phi_{j}\right\|_{L^{p}(\Omega)}<\varepsilon, \quad j \in\{1,2, \ldots, N\},
$$

and $\operatorname{supp} \Phi_{j} \subset \Omega \backslash B\left(a, \alpha_{j}\right)$. If

$$
R f(x)=\sum_{j=1}^{N} \beta_{j}(f) \Phi_{j}(x),
$$

then it is evident that $R \in F_{r}\left(X, L^{p}(\Omega)\right)$ and also

$$
\|P f-R f\|_{L^{p}(\Omega)} \leq \sum_{j=1}^{N}\left|\beta_{j}(f)\right|\left\|u_{j}-\Phi_{j}\right\|_{L^{p}(\Omega)} \leq c \varepsilon\|f\|_{X}
$$

for all $f \in X$. Let $\alpha=\min \left\{\alpha_{j}\right\}$. Then $\operatorname{supp} R f \subset \Omega \backslash B(a, \alpha)$.

\section{Main Results}

To prove the main results we need the following statement (see [18], Ch. 5, Section 4.6): 
Lemma 3.1. Let $u_{0}$ be the unit vector in $R^{n}$. Then by choosing $u=t u_{0}$, with $t$ fixed sufficiently large we can guarantee that

$$
|k(r(u+v))-k(r u)| \leq \frac{1}{2}|k(r u)|
$$

whenever $r \in R \backslash\{0\}$ and $|v| \leq 2$.

Lemma 3.2. Let $1<p<\infty$ and condition (2.3) be satisfied. Then from the boundedness of $K$ from $L_{w}^{p}\left(R^{n}\right)$ to $L_{v}^{p}\left(R^{n}\right)$ it follows that $w^{1-p^{\prime}}$ is locally integrable.

Proof. Suppose that $I(r):=\int_{B} w^{1-p^{\prime}}(x) d x=\infty$ for some positive $r$, where $B=B(0, r)$. Then there exists $g \in L^{p}(B), g \geq 0$, such that $\int_{B} w^{-1 / p} g=\infty$. Let us assume that $f_{r}(y)=g(y) w^{-1 / p}(y) \chi_{B}(y)$ and $B^{\prime}=B(r u, r)$, where $u=t u_{0}$ ( $t$ is from Lemma 3.1 and $u_{0}$ is the unit vector taken so that (2.3) holds). Obviously, $x=r u+u x^{\prime}$ for $x \in B^{\prime}$ and $y=r y^{\prime}$ for $y \in B$, where $\left|x^{\prime}\right|<1$ and $\left|y^{\prime}\right|<1$. Thus $x-y=r(u+v)$ with $|v|<2$ and consequently Lemma 3.1 yields $\left|K f_{r}(x)\right| \geq \frac{1}{2}\left(f_{r}\right)_{B}|k(r u)|$ for all $x \in B^{\prime}$. Hence by (2.3) the following estimates hold:

$$
\begin{gathered}
\left\|K f_{r}\right\|_{L_{v}^{p}\left(R^{n}\right)} \geq\left\|\chi_{B^{\prime}}(x) K f_{r}(x)\right\|_{L_{v}^{p}\left(R^{n}\right)} \geq \\
\geq \frac{b}{2 r t}\left(v_{B^{\prime}}\right)^{1 / p} f_{B}=\infty .
\end{gathered}
$$

On the other hand, $\left\|f_{r}\right\|_{L_{w}^{p}\left(R^{n}\right)}=\|g\|_{L^{p}(B)}<\infty$. Finally we conclude that $I(r)<\infty$ for all $r>0$.

Theorem 3.1. Let $1<p<\infty$. Suppose that conditions (2.1) $-(2.3)$ are satisfied. Then there exists no weight pair $(v, w)$ such that the singular integral operator $K$ is compact from $L_{w}^{p}\left(R^{n}\right)$ to $L_{v}^{p}\left(R^{n}\right)$. Moreover, if $K$ is bounded from $L_{w}^{p}\left(R^{n}\right)$ to $L_{v}^{p}\left(R^{n}\right)$, then the inequality

$$
\|K\|_{\mathcal{K}} \geq c \operatorname{ess}_{a \in R^{n}}\left(\frac{v(a)}{w(a)}\right)^{1 / p}
$$

holds, where the positive constant $c$ depends only on $n, t$ and $b$ (see Lemma 3.1 and $(2.3)$ ).

Let $K$ be bounded from $L_{w}^{p}\left(R^{n}\right)$ to $L_{v}^{p}\left(R^{n}\right)$. Suppose that

$$
K_{v} f(x)=v^{1 / p} K f(x) .
$$

Then it is easy to verify that

$$
\|K\|_{\mathcal{K}}=\operatorname{dist}\left\{K_{v}, \mathcal{K}\left(L_{w}^{p}\left(R^{n}\right), L^{p}\left(R^{n}\right)\right)\right\} .
$$

Let $\lambda>\|K\|_{\mathcal{K}}$. Then by Lemma 2.1 we have $\lambda>\alpha(K)$, where $\alpha(K):=$ $\operatorname{dist}\left\{K_{v}, F_{r}\right\}, F_{r}:=F_{r}\left(L_{w}^{p}\left(R^{n}\right), L^{p}\left(R^{n}\right)\right)$. Consequently there exists $P \in F_{r}$ for which

$$
\left\|K_{v}-P\right\|<\lambda .
$$


Let $a \in R^{n}$. Using Lemma 2.2 we find that there exist a positive number $\beta$ and $R \in F_{r}$ such that

$$
\|P-R\|<\frac{\lambda-\left\|K_{v}-P\right\|}{2}
$$

and supp $R f \subset R^{n} \backslash B(a, \beta)$ for all $f \in L_{w}^{p}\left(R^{n}\right)$. Hence

$$
\left\|K_{v}-R\right\| \leq \lambda \text {. }
$$

Thus the inequality

$$
\left\|\left(K_{v}-R\right) f\right\|_{L^{p}\left(R^{n}\right)} \leq \lambda\|f\|_{L_{w}^{p}\left(R^{n}\right)}
$$

holds for all $f \in L_{w}^{p}\left(R^{n}\right)$.

Let $B \equiv B(a, r)$, where $r<\beta$. Suppose that $B^{\prime}$ is the translation of $B$ in the direction of $u$, i.e. $B^{\prime}=B(a+r u, r)$, where $u=t u_{0}, t$ is taken so that the conditions of Lemma 3.1 are satisfied and $u_{0}$ is the unit vector chosen so that (2.3) holds. Let $f$ be any non-negative function supported in $B$. Consider $T f(x)$ for $x \in B^{\prime}$. We have

$$
K f(x)=\int_{B} k(x-y) f(y) d y
$$

with $x=a+r u+r x^{\prime},\left|x^{\prime}\right|<1$. Since $y \in B$, we find that $y=a+r y^{\prime}$ for $\left|y^{\prime}\right|<1$. Thus $x-y=r\left(u+r\left(y^{\prime}-x^{\prime}\right)\right)=r(u+v)$ with $|v|<2$. Further Lemma 3.1 and condition (2.3) yield

$$
|K f(x)| \geq \frac{1}{2} f_{B}|k(r u)| \geq c f_{B} \frac{1}{|B|},
$$

for all $x \in B^{\prime}$, where $|B|$ denotes a measure of $B$ and $c$ is the positive constant depending only on $n, b$ and $t$. Due to inequality (3.2) we obtain

$$
\int_{B^{\prime}} v(x)\left|\int_{B} k(x-y) f(y) d y\right|^{p} d x \leq \lambda^{p} \int_{B}(f(y))^{p} w(y) d y
$$

for all non-negative $f$ with supp $f \subset B$. Let $f(x)=w^{1-p^{\prime}}(x) \chi_{B}(x)$. Then using (3.3), we find that

$$
c^{p}\left(\int_{B^{\prime}} v(x) d x\right) f_{B}^{p} \frac{1}{|B|^{p}} \leq \lambda^{p} \int_{B} w^{1-p^{\prime}}(y) d y .
$$

Consequently by Lemma 3.2 we have

$$
c^{p} v_{B^{\prime}}\left(\left(w^{1-p^{\prime}}\right)_{B}\right)^{p-1} \leq \lambda^{p} .
$$

Further, observe that the equality

$$
\lim _{r \rightarrow 0} v_{B^{\prime}}=v(a)
$$

holds for almost all $a$. This follows from the obvious fact

$$
\left|v_{B^{\prime}}-v(a)\right| \leq \bar{c} \frac{1}{|\bar{B}|} \int_{\bar{B}}|v(x)-v(a)| d x \rightarrow 0
$$


as $r \rightarrow 0$, where $\bar{B}=B(a, r(t+1))$ and $\bar{c}$ is a positive constant.

Inequalities (3.4) and (3.5) yield

$$
c\left(\frac{v(a)}{w(a)}\right)^{1 / p} \leq \lambda
$$

for almost all $a$; here the positive constant $c$ depends only on $a, n$ and $t$. As $\lambda$ is an arbitrary number greater than $\|K\|_{\mathcal{K}}$, we conclude that (3.1) holds.

An analogous result for maximal functions was derived in [7].

We recall that $\Gamma$ is called a smooth curve if $t^{\prime}(s)$ is continuous (and in the case of its closedness, $t^{\prime}(0)=t^{\prime}(l)$ ). For smooth curves the boundedness of $S_{\Gamma}$ in $L^{p}(\Gamma), 1<p<\infty$, was derived in [1]. In [15] (see also [14], pp. 55-56) it was proved that $S_{\Gamma}$ is bounded in $L_{w}^{p}(0, l)$ if and only if $w \in A_{p}(0, l)$.

Theorem 3.2. Let $1<p<\infty$. Suppose that $\Gamma$ is a Jordan smooth curve. Then there exists no weight pair $(v, w)$ such that the singular integral operator $S_{\Gamma}$ is compact from $L_{w}^{p}(0, l)$ to $L_{v}^{p}(0, l)$. Moreover, if $S_{\Gamma}$ is bounded from $L_{w}^{p}(0, l)$ to $L_{v}^{p}(0, l)$, then the inequality

$$
\left\|S_{\Gamma}\right\|_{\mathcal{K}} \equiv \operatorname{dist}\left\{S_{\Gamma}, \mathcal{K}\left(L_{w}^{p}(0, l), L_{v}^{p}(0, l)\right)\right\} \geq \frac{1}{4 \pi} \operatorname{ess} \sup \left(\frac{v(a)}{w(a)}\right)^{1 / p}
$$

holds.

Proof. Let $S_{\Gamma}$ be bounded from $L_{w}^{p}(0, l)$ to $L_{v}^{p}(0, l)$ and $a \in(0, l)$. Then, using Lemmas 2.1 and 2.2 , there exist a positive number $\beta$ and $R \in$ $F_{r}\left(L_{w}^{p}(0, l), L_{v}^{p}(0, l)\right)$ such that

$$
\left\|\left(S_{\Gamma, v}-R\right) f\right\|_{L^{p}(0, l)} \leq \lambda\|f\|_{L_{w}^{p}(0, l)}, \quad f \in L_{w}^{p}(0, l),
$$

and supp $R f \subset(0, l) \backslash I(a, \beta)$, where

$$
S_{\Gamma, v} f(x)=v^{1 / p}(x) S_{\Gamma} f(x),
$$

$I(a, \beta)=(a-\beta, a+\beta)$ and $\lambda>\left\|S_{\Gamma}\right\|_{\mathcal{K}}$. Further, let $I_{1}:=(a-r, a)$, $I_{2}:=(a, a+r)$, where $r<\beta$. Suppose that $\varphi(s)=f(t(s))$ is a non-negative function with $\operatorname{supp} \varphi \subset I_{2}$. Then (see [15], [14], p.56)

$$
\left|S_{\Gamma} f(t(\sigma))\right| \geq \frac{1}{2 \pi} \int_{I_{2}} \frac{\varphi(s)}{s-\sigma} d s \geq \frac{1}{4 r \pi} \int_{I_{2}} \varphi(s) d s
$$

for $\sigma \in I_{1}$ and sufficiently small $r$. Thus we have

$$
\left|S_{\Gamma} f(t(\sigma))\right| \geq\left(\frac{1}{4 r \pi} \int_{I_{2}} \varphi(s) d s\right) \chi_{I_{1}}(\sigma)
$$

for any $\sigma$. Taking into account inequality (3.8) and the proof of Lemma 3.2 we have that $w^{1-p^{\prime}}$ is locally integrable. Let $\varphi(s)=w^{1-p^{\prime}}(s) \chi_{I_{2}}(s)$. Then 
by (3.7) we arrive at a conclusion that

$$
\frac{1}{(4 \pi)^{p}}\left(\frac{1}{r} \int_{a}^{a+r} w^{1-p^{\prime}}(s) d s\right)^{p-1}\left(\frac{1}{r} \int_{a-r}^{a} v(s) d s\right) \leq \lambda^{p}
$$

holds for all $a \in(0, l)$. The latter inequality yields (3.6).

Remark. From the proof of Theorem 3.2 it easily follows that for the Hilbert transform $H$ the constant $c$ is equal to $\frac{1}{2 \pi}$.

Acknowledgements. The research was partially supported by Grant No. 1.7 of the Georgian Academy of Sciences. I express my gratitude to Prof. V. Kokilashvili for drawing my attention to the problems considered in this paper and helpful remarks.

\section{References}

[1] A. P. Calderón, Cauchy integrals on Lipschitz curves and related operators, Proc. Nat. Acad. Sci. USA, 74(1977), No. 4, 1324-1327.

[2] R. R. Coifman and Ch. Fefferman, Weighted norm inequalities for maximal functions and singular integrals, Studia Math., 51(1974), 241250.

[3] D. E. Edmunds and W.D. Evans, Spectral Theory and Differential Operators, Oxford Univ. Press, Oxford, 1987.

[4] D. E. Edmunds and V. Kokilashvili, Two-weight inequalities for singular integrals, Canadian Math. Bull, 38(1995), 119-125.

[5] D. E. Edmunds, V. Kokilashvili and A. Meskhi, Two-weight estimates for singular integrals defined on spaces of homogeneous type, Canad. J. Math., 52(2000), No. 3, 468-502.

[6] D. E. Edmunds, V. Kokilashvili and A. Meskhi, Bounded and Compact Integral Operators, Kluwer Academic Publishers, Dordrecht, Boston, London, 2002.

[7] D. E. Edmunds and A. Meskhi, On a measure of non-compactness for maximal operators, Math. Nachr., 254-255 (2003), 97-106.

[8] I. Feldman, N. Krupnik and I. Spitkovsky, Norms of the singular integral operators with Cauchy kernel along certain contours, Integral Equations Operator Theory, 24(1996), 68-80.

[9] T. Garcia-Guerva and T. Rubio de Francia, Weighted Norm Inequalities and Related Topics, North Holland, Amsterdam, 1985.

[10] I. Genebashvili, A. Gogatishvili, V. Kokilashvili and M. Krbec, Weight theory for integral transforms on spaces of homogeneous type, Pitman Monographs and Surveys in Pure and Applied Mathematics, 92, Longman, Harlow, 1998. 
[11] I. Gohberg and N. Krupnik, On the spectrum of singular integral operators on the spaces $L_{p}$, (Russian) Studia Math., 31(1968), 347362.

[12] I. Gohberg and N. Krupnik, On the norm of the Hilbert transform on the space $L_{p}$, (Russian) Funktsional Anal. i. Prilozh. 2(1968), No. 2, 91-92.

[13] R. A. Hunt, B. Muckenhoupt and R. Wheeden, Weighted norm inequalities for the conjugate function and Hilbert transform, Trans. Amer. Math. Soc., 176(1973), 227-251.

[14] G. Khuskivadze, V. Kokilashvili and V. Paatashvili, Boundary value problems for analytic and harmonic functions in domains with nonsmooth boundaries, Applications to conformal mappings, Memoirs on Differential Equations and Math. Phys., 14(1998), 1-195.

[15] V. Kokilashvili, On singular and bisingular integral operators in weighted spaces (Russian), Proc. Mat. Inst. Razmadze Akad. Nauk Gruzin SSR, 19(1982), 51-79.

[16] N. Krupnik and I. Verbitsky, Exact constants in the theorems of $K$. I. Babenko and B. V. Khvedelidze on the boundedness of singular operators (Russian), Soobshch. AN Gruz. SSR, 85(1977), No. 1, 2124.

[17] S. K. Pichorides, On the best values of the constants in the theorems of M. Riesz, Zygmund and Kolmogorov, Studia Math., 44(1972), 165-179.

[18] E. M. Stein, Harmonic Analysis: Real Variable Methods, Orthogonality and Oscillatory Integrals, Princeton University Press, Princeton, New Jersey, 1993.

A. Razmadze Mathematical Institute

Georgian Academy of Sciences

1, M. Aleksidze St

380093 Tbilisi

Georgia

(Email : meskhi@rmi.acnet.ge) 


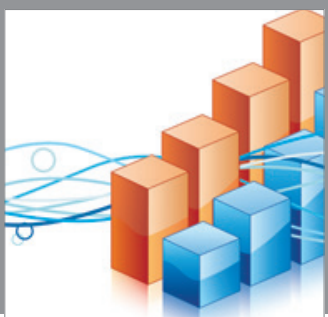

Advances in

Operations Research

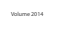

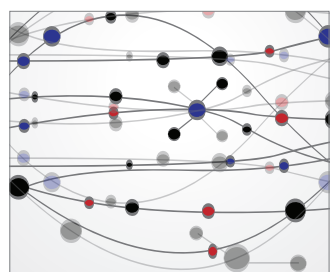

\section{The Scientific} World Journal
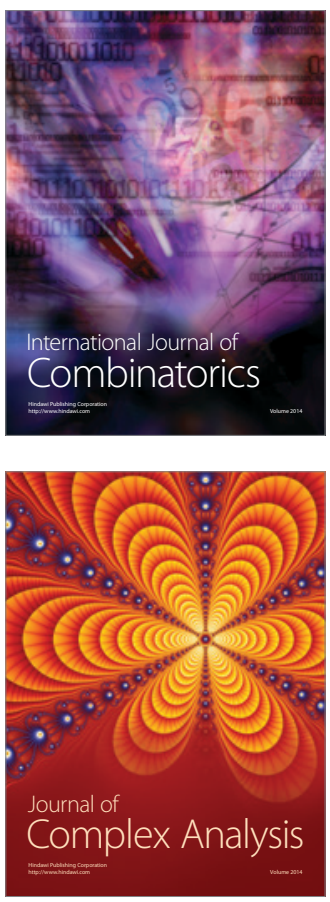

International Journal of

Mathematics and

Mathematical

Sciences
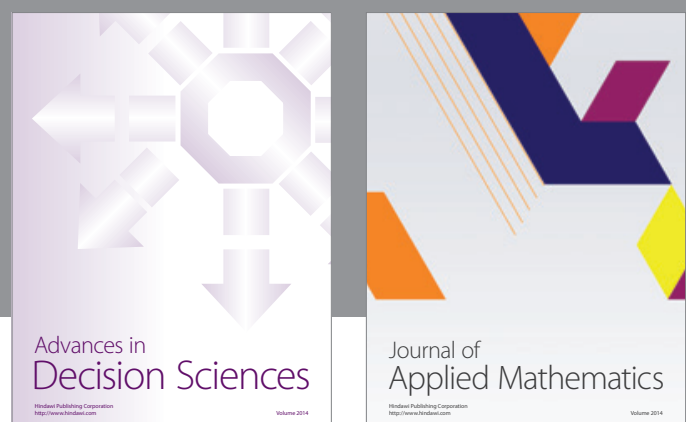

Journal of

Applied Mathematics
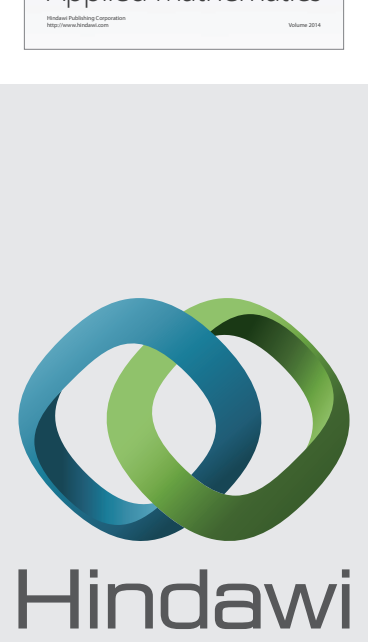

Submit your manuscripts at http://www.hindawi.com
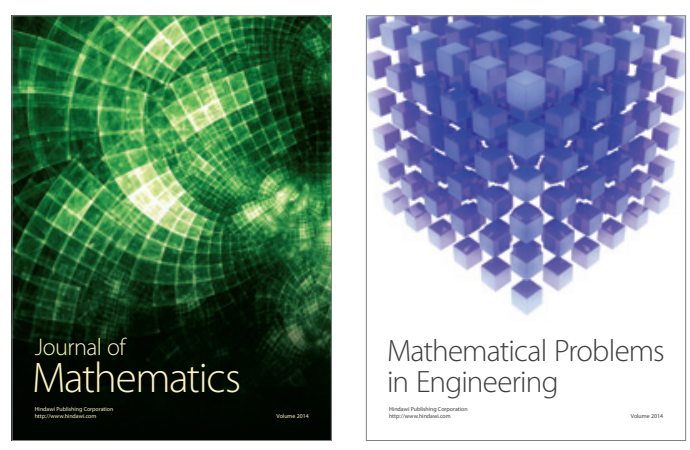

Mathematical Problems in Engineering
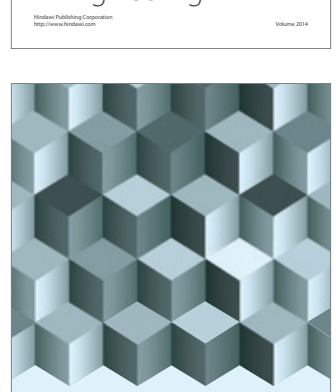

Journal of

Function Spaces
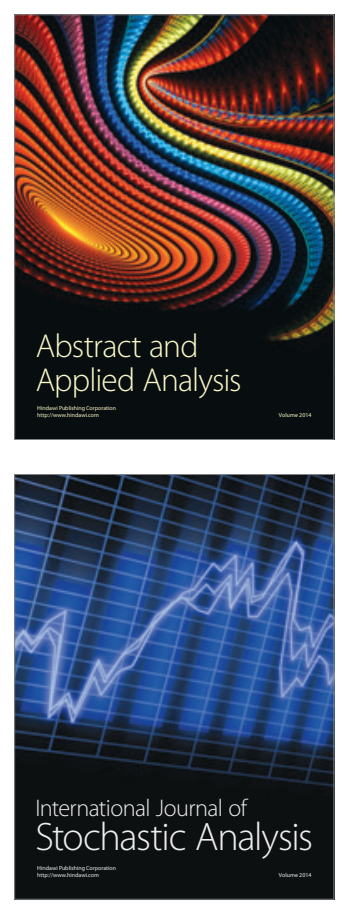

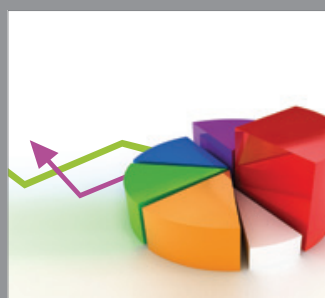

ournal of

Probability and Statistics

Promensencen
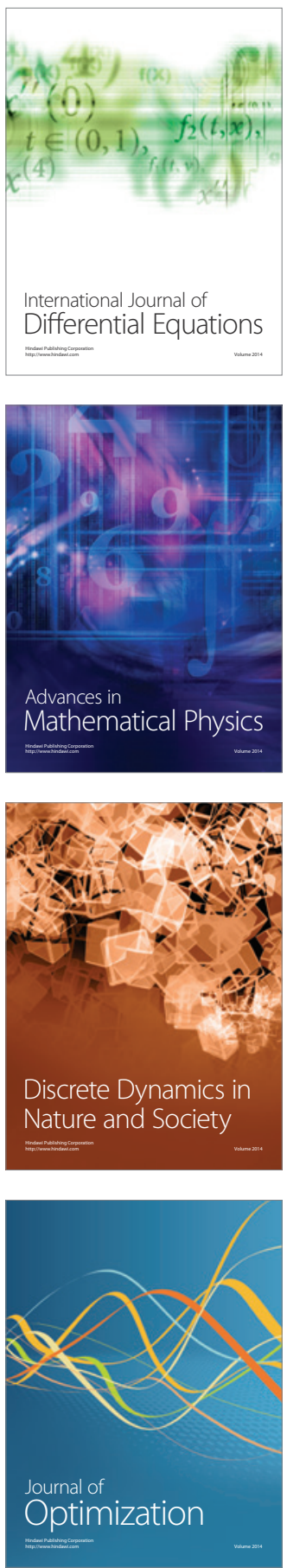